\title{
The quest to improve outcomes for abdominal wall incisional hernia repair in Pietermaritzburg: between Scylla and Charybdis
}

\author{
A Gurunand, ${ }^{1,2}$ (i) MTD Smith, ${ }^{1,2}$ (i) JL Bruce, ${ }^{1,2}$ (i) VY Kong, ${ }^{2,3}$ (D) GL Laing, ${ }^{2}$ (i) V Govindasamy, ${ }^{1}$ (i) DL Clarke $e^{1,2,3}$ \\ ${ }^{1}$ Department of General Surgery and Trauma, Grey's Hospital, South Africa \\ ${ }^{2}$ Department of Surgery, University of KwaZulu-Natal, South Africa \\ ${ }^{3}$ Department of Surgery, University of the Witwatersrand, South Africa
}

Corresponding author, email: avi.gurunand@gmail.com

Background: The repair and outcomes of incisional abdominal wall hernias have not yet been benchmarked to allow comparison with recommended best practice in a South African context. This study aimed to address this deficit.

Method: Patients who underwent an incisional hernia repair between December 2012 and December 2018 were analysed in respect to the following variables: demographics, comorbidities, indication for surgery, site, size, surgical approach, mesh usage, operating times, complications and 30-day mortality.

Results: Of the cohort of 224 patients, 185 underwent elective repair. There were 152 open and 72 laparoscopic procedures, and 17 patients (8\%) required a repeat operation with an overall in-hospital mortality rate of 6\% (13). Eight patients developed an enteric leak. There were nine cardiovascular complications, 24 respiratory complications, 22 surgical site infections and 13 patients developed an acute kidney injury (AKI). There were 39 emergency operations. The emergency cohort were older than the elective with a higher rate of cardiovascular or surgical (CVS) complications and AKI. Eight patients developed an enteric leak. Mortality rates of were significantly higher in the emergency operation cohort compared to the elective group (18\% vs 3\%). The 13 in-hospital deaths were older, more likely to have undergone an emergency operation, to be diabetic ( $46 \%$ vs $10 \%)$, hypertensive ( $92 \%$ vs $33 \%)$, have a bowel anastomosis ( $39 \%$ vs $9 \%$ ), experience an enteric leak ( $46 \%$ vs $1 \%$ ) and require repeat operation than the patients who survived.

Conclusion: Incisional abdominal wall hernias are difficult to manage as the patients often have several comorbidities which when coupled with an emergency operation leads to poor outcomes. Improving outcomes requires strategies that address comorbidities and shift the focus to elective rather than emergency repair.

Keywords: incisional, hernia

\section{Introduction}

There have been dramatic advances in the surgical management of incisional abdominal hernias over the last twenty years. The advent of laparoscopy, the rapid progress in mesh technology, the development of clinical algorithms, the concept of a multidisciplinary team and enhanced postsurgical recovery programmes have all impacted on the practice of hernia surgery. ${ }^{1,2}$ Despite this, surgical repair of incisional abdominal wall hernias remains challenging. This is because of the associated comorbidity as well as the attenuated nature of the abdominal wall. In both Europe and North America, there has been a realisation that abdominal incisional hernia repair must be performed by surgeons and institutions which have a degree of consolidated expertise in, and experience with, incisional abdominal wall hernia repair. ${ }^{1-3}$ South Africa has been slow to adopt such an approach. There is now an established Hernia Interest Group, which has published guidelines which include the management of ventral and incisional abdominal wall hernias. ${ }^{4}$ Although the department of surgery in Pietermaritzburg has been developing a sub-specialist driven model of surgical care and surgical training over the last decade, this has not been applied to the management of abdominal wall hernias. Just as the hero of the Trojan wars, Odysseus, had to steer between the twin threats of Scylla and Charybdis, so some surgeons try to avoid surgery and adopt an expectant attitude towards patients with incisional hernias, as they feel the risk of elective repair outweighs the risk of observation. The department has a quality improvement programme (QIP) type approach to development. This involves a continuous audit of outcomes and introspection followed by strategic planning based on the data. This is facilitated by the Hybrid Electronic Medical Registry (HEMR), which captures data on all surgical patients in the hospital. Ongoing departmental strategic review has identified the management of abdominal wall hernias as a potentially neglected pathology in the department which could benefit from a more focused approach. This study stems from this internal QIP in the department. It goes on to describe and quantify our experience with the management of abdominal 
wall hernias and to review our postoperative outcomes. The data is intended to be used to structure algorithms within our current constraints, for the management these hernias.

\section{Clinical setting}

The Department of Surgery, at Grey's Hospital, provides tertiary surgical care to the city of Pietermaritzburg and the western third of the province, a population of about three million. The department maintains an electronic database called the HEMR, which captures data on all surgical admissions. Although the department has sub-specialist teams for trauma and burns, breast and endocrine, uppergastrointestinal and lower-gastrointestinal surgery, there are a number of pathologies which resist easy classification into one of the above divisions. These include the management of incisional abdominal wall hernias. Currently, all hernias are reviewed in a general clinic and are operated on by individual teams led by consultants with a specialised interest in a field other than incisional abdominal wall hernia repair. This has led to a heterogeneous approach rather than a best practice approach as recommended by the guidelines.

\section{Methods}

All patients who had undergone an incisional abdominal wall hernia repair between December 2012 and December 2018 were identified using a word search of the database. Patient demographics, indication (elective vs emergency), type and approach of hernia repair, the use of prosthetic mesh, comorbid profile, operating times, in-patient adverse events and 30-day in-patient mortality rates were extracted. Hernias were further subclassified based on size of defect with those with a defect of over $10 \mathrm{~cm}$ (so-called large incisional hernias or LIH) classified separately and similar outcomes analysed. Locally these are often termed ventral hernias and arise following an open abdomen. This classification is in line with European Hernia Society Guidelines. ${ }^{4}$

\section{Statistics}

Descriptive statistics were calculated for age, comorbid risk factors, surgical approach and type of hernia repair, the use of prosthetic mesh, operating times, in-patient adverse events as well as 30-day in-patient mortality rates. Central tendency was expressed as medians and interquartile ranges (IQRs). Comorbidities and risk factors were expressed as frequencies and percentages of the total sample. Those who underwent emergent surgery were compared to those who underwent elective repair. Survivors and non-survivors were similarly compared. When odds ratios (ORs) were calculated, females and non-survivors were used as reference values.

\section{Results}

\section{Demographic details}

During the period under review, 224 patients of which 59 $(26 \%)$ were male with a mean age of 47.2 (15.7) years underwent operative repair of an incisional abdominal wall hernia. Eighty-three per cent (185) of patients underwent an elective repair. A comparison of demographic, comorbid variables and hernia characteristics by sex is shown in Table I. Females were older than the males with median ages of 49

\begin{tabular}{|c|c|c|c|c|c|c|c|}
\hline \multirow{2}{*}{ Variables } & \multicolumn{2}{|c|}{ Male } & \multicolumn{2}{|c|}{ Female } & \multicolumn{2}{|c|}{ All } & \\
\hline & \multicolumn{2}{|c|}{$n=59$} & \multicolumn{2}{|c|}{$n=165$} & \multicolumn{2}{|c|}{$n=224$} & $p$ \\
\hline Age median (IQR) & \multicolumn{2}{|c|}{$38(31-48)$} & \multicolumn{2}{|c|}{$49(39-62)$} & \multicolumn{2}{|c|}{$45(35-57)$} & $<0.001$ \\
\hline Operative time & \multicolumn{2}{|c|}{$120(82-165)$} & \multicolumn{2}{|c|}{$90(64-120)$} & \multicolumn{2}{|c|}{$95(70-135)$} & $<0.001$ \\
\hline Comorbidities & $n$ & $\%$ & $n$ & $\%$ & $n$ & $\%$ & \\
\hline HPT & 8 & 13 & 73 & 45 & 81 & 36 & $<0.001$ \\
\hline DM & 4 & 7 & 23 & 14 & 27 & 12 & 0.122 \\
\hline COPD & 0 & 0 & 8 & 5 & 8 & 4 & 0.111 \\
\hline RVD & 11 & 18 & 37 & 23 & 48 & 21 & 0.449 \\
\hline IHD & 4 & 7 & 2 & 1 & 6 & 3 & 0.048 \\
\hline CKD & 0 & 0 & 2 & 1 & 2 & 1 & 1 \\
\hline DYS & 1 & 2 & 5 & 3 & 6 & 3 & 1 \\
\hline Obesity & 17 & 28 & 78 & 48 & 95 & 42 & 0.007 \\
\hline \multirow{2}{*}{ Hernia } & \multicolumn{2}{|c|}{ Male } & \multicolumn{2}{|c|}{ Female* } & \multicolumn{2}{|c|}{ All } & \\
\hline & $n$ & $\%$ & $n$ & $\%$ & $n$ & $\%$ & $p$ \\
\hline Elective repair & 53 & 87 & 132 & 81 & 185 & 83 & 0.300 \\
\hline Incisional $<10 \mathrm{~cm}$ & 20 & 33 & 144 & 88 & 164 & 73 & $<0.001$ \\
\hline Hernia size $>10 \mathrm{~cm}$ & 41 & 67 & 19 & 12 & 60 & 27 & \\
\hline Midline & 60 & 98 & 77 & 47 & 137 & 61 & $<0.001$ \\
\hline Other & 3 & 5 & 87 & 53 & 90 & 40 & \\
\hline Mesh used & 11 & 18 & 100 & 61 & 111 & 50 & $<0.001$ \\
\hline Laparoscopic & 7 & 12 & 64 & 39 & 71 & 32 & $<0.001$ \\
\hline Open & 54 & 89 & 99 & 61 & 153 & 68 & \\
\hline
\end{tabular}

HPT - hypertension, DM - diabetes, COPD - chronic obstructive pulmonary disease, RVD - retroviral disease, IHD - ischaemic heart disease, CKD - chronic kidney disease, DYS - dyslipidaemia 
years (IQR 39-62) and 38 years (IQR 31-48) respectively. This finding was statistically significant $(p<0.001)$. Approximately $42 \%$ (95) of all patients were obese with a higher proportion of female (48\%) as compared to male patients (28\%). Over one-third of patients were hypertensive and this was significantly higher in the female cohort $(45 \%$ vs $13 \%, p<0.001)$. Twelve per cent of patients (27) were diabetic. Elective operations significantly outnumbered emergencies with 185 (83\%) elective procedures and 39 (17\%) emergency operations.

\section{Hernia characteristics by sex}

Seventy-three per cent (164) hernias were less than $10 \mathrm{~cm}$ in size. Females were more likely to have small hernias $(144 ; 88 \%)$ than males $(20 ; 33 \%)$. Sixty-one per cent (137) of hernias were repaired using a midline laparotomy. A midline approach was more likely to be associated with males. A midline approach was used in $98 \%$ of males, and $53 \%$ of women had a pfannenstiel incision. The male sex was predictive of a midline approach (OR 0.02; 95\% $\mathrm{CI}<0.001-0.08)$ and hernias $>10 \mathrm{~cm}(\mathrm{OR} 0.66 ; 95 \% \mathrm{CI}$ $0.03-0.13)$. A prosthetic mesh was used in approximately $50 \%$ (111) of cases. The odds of using a mesh in females was more than 7 times that of males (95\% CI 3.53-15.36). Females had almost 5 times the odds of having laparoscopic surgery than males (OR 4.87; 95\% CI 2.20-12.47).

\begin{tabular}{lccc}
\multicolumn{4}{c}{ Table II: A comparison of emergency and elective cases } \\
\hline & $\begin{array}{c}\text { Emergency } \\
\boldsymbol{n}=\mathbf{3 9}\end{array}$ & $\begin{array}{c}\text { Elective } \\
\boldsymbol{n}=\mathbf{1 8 5}\end{array}$ & $\boldsymbol{p}$ \\
\hline Age & $57(47-68)$ & $43(34-56)$ & $<0.001^{*}$ \\
Operative time & $93(73-127)$ & $95(70-135)$ & 0.817 \\
Laparoscopy & $5(13 \%)$ & $66(36 \%)$ & 0.005 \\
Comorbidities & & & \\
\hline DM & $8(21 \%)$ & $19(10 \%)$ & 0.074 \\
HPT & $21(54 \%)$ & $60(32 \%)$ & 0.011 \\
RVD & $8(21 \%)$ & $40(22 \%)$ & 0.878 \\
IHD & $2(5 \%)$ & $4(2 \%)$ & 0.281 \\
CKD & 0 & $2(1 \%)$ & 1 \\
COPD & $3(8 \%)$ & $5(3 \%)$ & 0.146 \\
Complications & & & \\
\hline Leak & $4(10 \%)$ & $4(2 \%)$ & 0.033 \\
SSI & $7(18 \%)$ & $15(8 \%)$ & 0.061 \\
AKI & $7(18 \%)$ & $6(3 \%)$ & $<0.001$ \\
\hline
\end{tabular}

HPT - hypertension, DM - diabetes, COPD - chronic obstructive pulmonary disease, RVD - retroviral disease, IHD - ischaemic heart disease, CKD - chronic kidney disease, DYS - dyslipidaemia

\section{Comparison between emergency and elective hernia repairs}

Patients undergoing emergency repair were older than those undergoing elective repairs $(p<0.001)$. Emergency patients had a median age of 57 years (IQR 47-68) and electives had a median age of 43 years (34-56). Thirty-six per cent (66)

Table III: Comparison of demographic, hernia variables, complications and comorbidities between survivors and non-survivors

\begin{tabular}{|c|c|c|c|c|c|}
\hline \multirow{2}{*}{ Age (median; IQR) } & \multicolumn{2}{|c|}{ Survivors $n=211$} & \multicolumn{2}{|c|}{ Non-survivors $n=13$} & \multirow{2}{*}{$\begin{array}{c}p \\
<0.001\end{array}$} \\
\hline & \multicolumn{2}{|c|}{$44(34-56)$} & \multicolumn{2}{|c|}{$68(59-76)$} & \\
\hline Operation time (median; IQR) & 90 & $70-130$ & 130 & $105-185$ & 0.008 \\
\hline Hernia variables & $n$ & $\%$ & $n$ & $\%$ & \\
\hline Elective & 179 & 85 & 6 & 46 & $<0.001$ \\
\hline$>10 \mathrm{~cm}$ & 58 & 27 & 2 & 15 & 0.339 \\
\hline Laparoscopic & 70 & 33 & 1 & 8 & 0.055 \\
\hline Mesh & 104 & 49 & 7 & 54 & 0.750 \\
\hline Anastomosis & 19 & 9 & 5 & 39 & $<0.001$ \\
\hline \multicolumn{6}{|l|}{ Complications } \\
\hline Enteric leak & 2 & 1 & 6 & 46 & $<0.001$ \\
\hline SSI & 17 & 8 & 5 & 39 & 0.001 \\
\hline Iatrogenic injury & 14 & 7 & 4 & 31 & 0.013 \\
\hline Repeat operation & 9 & 4 & 9 & 69 & $<0.001$ \\
\hline \multicolumn{6}{|l|}{ Comorbidities } \\
\hline HPT & 69 & 33 & 12 & 92 & $<0.001$ \\
\hline $\mathrm{DM}$ & 21 & 10 & 6 & 46 & $<0.001$ \\
\hline COPD & 8 & 4 & 0 & 0 & 1 \\
\hline RVD & 47 & 22 & 1 & 8 & 0.308 \\
\hline IHD & 5 & 2 & 1 & 8 & 0.304 \\
\hline CKD & 1 & $<1$ & 1 & 8 & 0.113 \\
\hline DYS & 6 & 3 & 0 & 0 & 1 \\
\hline
\end{tabular}

HPT - hypertension, DM - diabetes, COPD - chronic obstructive pulmonary disease, RVD - retroviral disease, IHD - ischaemic heart disease, CKD - chronic kidney disease, DYS - dyslipidaemia 
of elective repairs and only 13\% (5) of emergencies were performed laparoscopically. Elective surgery was associated with laparoscopic approach with an OR of 3.66 (95\% CI 1.47-11.28). Both groups had similar comorbidity profiles. However, elective cases were less likely than emergency cases to have chronic hypertension (OR $0.41 ; 95 \%$ CI 0.20-0.84). Operative times were similar between both groups $(p=0.817)$ with median emergency operative times of 93 minutes (IQR 73-127) and median elective operative time of 95 minutes (IQR 70-135). With regards to surgical complications, elective surgeries were less likely to be complicated by enteric leaks and AKI with ORs of $0.2(95 \%$ CI $0.04-0.90)$ and 0.16 (95\% CI 0.05-0.51) respectively. Rates of surgical site sepsis were not significantly different between the two groups. Details can be seen in Table II.

There was a higher rate of CVS complications and AKI in the emergency cohort with a significantly higher mortality rate overall and when compared to those having elective surgery.

\section{Comparison between survivors and non-survivors}

The overall mortality rate was $6 \%$ (13). Age, operative time and emergency surgery were all associated with mortality. Non-survivors tended to be older than survivors (OR 1.09; $95 \%$ CI 1.05-1.14). The median age of survivors was 44 years (IQR 34-56) and the median age of non-survivors was 68 years (IQR 59-76). Longer operative times were associated with mortality (OR 1.01; 95\% CI 1.002-1.018). Elective surgery was inversely associated with mortality (OR 0.16; 95\% CI 0.05-0.51). Hernia size, laparoscopic vs open approach and use of a mesh were not found to be associated with mortality. If an anastomosis was performed, the odds of mortality were increased more than 6 times (OR 6.28; 95\% CI 1.70-21.27) (Table III).

Enteric leak, surgical site infection (SSI), iatrogenic injury and repeat operation were all associated with mortality. Eight patients sustained an enteric leak following initial surgery. Suffering an enteric leak increased the odds mortality by almost 80 times (OR 78.90; 14.81-691.39). Repeat operation increased the odds of mortality by approximately 47 times (OR 46.80; 95\% CI 12.61-208.43). Further details may be seen in Table III. Hypertension and diabetes mellitus were also associated with mortality with an odds ratios of 21.62 (95\% CI 4.10-535.93) and 7.65 (95\% CI 2.22-25.76) respectively.

\section{Discussion}

This audit has benchmarked our outcomes for abdominal wall incisional hernia repair and this will hopefully allow for comparison when quality improvement initiatives are undertaken. Several issues stand out. Patients with an incisional abdominal wall hernia are a high-risk cohort with multiple comorbidities. ${ }^{3,5-8}$ As evidenced by the fact that just under half $(42 \%)$ of all patients in this series were obese, over one third were hypertensive and $12 \%$ were diabetic. Interventions to improve and modify these comorbidities must be instituted.4,8 These should include weight loss, cessation of smoking, preoperative fitness training as well as optimisation of medical treatment of comorbidities. ${ }^{10}$ This will require close cooperation with a number of other specialities as well as with the allied health practitioners. ${ }^{8,9,11}$ In many ways, incisional abdominal wall hernia surgery represents an extreme aspect of the sub-specialisation debate in that they represent a specific disease process, rather than a more general organ system in need of systematic subspecialist care. ${ }^{2,9,12}$

Little is known about surgical outcomes of hernia repair from comparable countries to South Africa. A report from Pakistan ${ }^{7}$ identified a number of risk factors for short-term morbidity and mortality. These included comorbidities such as diabetes mellitus, emergency surgery, intraoperative contamination as well as recurrent hernias. This is similar to our findings. Reports from high-income countries document variable mortality rates, ranging from $0.4-10.4 \%$ and high rates of morbidity, related especially to wound and pulmonary complications. , $^{7-11}$ In addition to concerns around patient comorbidities, the surgery itself is technically demanding as evidenced by the high rate of surgical misadventure and iatrogenic enteric injury in our series. Iatrogenic enteric injury is associated with morbidity and mortality. ${ }^{8,9}$ Large incisional hernias have complex anatomy and are challenging to repair, consequently numerous techniques of repair have been described. These include component separation, aponeuroplasty and abdominal wall reconstruction using composite meshes. ${ }^{8,14}$ All these techniques are technically challenging and formal training in these techniques is probably necessary to improve outcomes. . $^{3,12-14}$ Surgical expertise must be appropriate to the level of complexity, and it is apparent that these operations should not be considered as training operations. Surgical algorithms may well help standardise techniques and approaches. ${ }^{4,8,14}$ There is little to suggest that we have managed to do this in our setting. Channelling this surgery to a defined group of surgeons will help develop the appropriate skill set amongst them. Our overall results reflect this lack of centralisation, with a mortality rate of nine per cent.

Despite the challenges elucidated, a surgical approach remains preferable to non-operative strategies. ${ }^{6}$ Our data clearly supports the contention that incisional abdominal wall hernias need to be operated on and should not be treated expectantly. Non-operative treatment often results in the patient presenting with an acute surgical complication which mandates surgery. 2,9,11,12 Emergency surgery for an incisional abdominal wall hernia is significantly more likely to result in morbidity and even mortality.,7,9 This is clearly shown in our results with significantly higher mortality and morbidity rates in the emergency cohort. The data from this review suggests that deferring the operation often results in a high rate of presentation with acute complications, mandating emergency surgery. Emergency repair and an enteric leak are associated with significantly increased morbidity and mortality rates. ${ }^{9,11}$ Unlike Odysseus, we cannot simply steer a middle path between the Scylla of non-operative management and the Charybdis of a surgical approach. The best analogy is obesity surgery. ${ }^{10}$ Although medical management of morbid obesity is ineffectual, surgical treatment requires a comprehensive multidisciplinary system, if it is to be undertaken with reasonable outcomes. This surgical approach to abdominal wall hernias must be comprehensive and systematic and implement the lessons of obesity surgery. ${ }^{7,11}$

\section{Conclusion}

Incisional abdominal wall hernias are difficult to manage as the patients have numerous comorbidities which need to be aggressively addressed. In addition, the surgery is 
technically challenging. Aggressive attempts to improve the surgical outcome for this group of patients are essential as non-operative expectant management is associated with emergency presentation and even worse outcomes.

\section{Conflict of interest}

The authors do not have any relevant disclosures or conflicts of interest for this work

\section{Funding source}

No funding was required.

\section{Ethical approval}

Ethical approval for the maintenance of our registry and for this study was formally approved by the Biomedical Research Ethics Committee of the University of KwaZuluNatal (Reference number: BCA 207/09 and BCA 221/13).

\section{ORCID}

A Gurunand (iD https://orcid.org/0000-0003-0477-4663

MTD Smith (iD https://orcid.org/0000-0002-6954-153X

JL Bruce (iD) https://orcid.org/0000-0001-8666-4104

VY Kong (iD https://orcid.org/0000-0003-2291-2572

GL Laing (iD) https://orcid.org/0000-0001-8075-0386

V Govindasamy (iD https://orcid.org/0000-0001-8232-4882

DL Clarke (iD https://orcid.org/0000-0002-8467-1455

\section{REFERENCES}

1. Rutkow IM. Demographic and socioeconomic aspects of hernia repair in the United States in 2003. Surg Clinorth Am. 2003;83(5):1045-51. https://doi.org/10.1016/S00396109(03)00132-4 .

2. Poulose BK, Shelton J, Phillips S, et al. Epidemiology and cost of ventral hernia repair: making the case for hernia research. Hernia. 2012;16(2):179-83. https://doi.org/10.1007/ s10029-011-0879-9.

3. Zahiri HR, Belyansky I, Park A. Abdominal wall hernia. Curr Probl Surg. 2018;55:286-317. https://doi.org/10.1067/j. cpsurg.2018.08.005.
4. Bougard H, Coolen D, De Beer R, et al. HIG (SA) Guidelines for the management of ventral hernias. S Afr J Surg. 2016;4(4):S1-S32.

5. Pillay $Y$, Naidoo N, Madiba TE. Incisional hernia: experience in a single surgical unit. East Cent Afr J Surg. 2007;12(1):12.

6. Helgstrand F, Rosenberg J, Kehlet H, Bisgaard T. Outcomes after emergency versus elective ventral hernia repair: a prospective nationwide. World J Surg. 2013;37:2273-9. https://doi.org/10.1007/s00268-013-2123-5.

7. Memon AA, Khan A, Zafar H, Murtaza G, Zaidi M. Repair of large and giant incisional hernia with onlay mesh: perspective of a tertiary care hospital of a developing country. Int J Surg. 2013;11:41-e45. https://doi.org/10.1016/j.ijsu.2012.11.006.

8. Faylona JM. Evolution of ventral hernia repair. Asian J Endosc Surg. 2017;10(3):252-8. https://doi.org/10.1111/ases.12392.

9. Pereira AJ, Montcusi B, López-Cano M, Hernández-Granados P, Fresno de Prado L. Risk factors for bad outcomes in incisional hernia repair: lessons learned from the National Registry of Incisional Hernia (EVEREG). Cir ESP. 2018;96(7):436-42. https://doi.org/10.1016/j.ciresp.2018.03.008.

10. Loots E, Sartorius B, Paruk IM, Clarke DL. The successful implementation of a modified enhanced recovery after surgery (ERAS) program for bariatric surgery in a South African teaching hospital. Surg Laparosc Endosc Percutan Tech. 2018;28(1):26-29. https://doi.org/10.1097/ SLE.0000000000000488.

11. Deerenberg EB, Timmermans L, Hogerzeil DP, et al. A systematic review of the surgical treatment of large incisional hernia. Hernia. 2015;19:89-101. https://doi.org/10.1007/ s10029-014-1321-x.

12. Sartelli M, Coccolini F, Van Ramshorst GH, et al. WSES guidelines for emergency repair of complicated abdominal wall hernias. World J Emerg Surg. 2013;50:8. https://doi. org/10.1186/1749-7922-8-50.

13. White TJ, Santos MC, Thompson JS. Factors affecting wound complications in repair of ventral hernias. Am Surg. 1998;64(3):276-80.

14. Otero J, Huber AT, Heniford BT. Laparoscopic hernia repair. Adv Surg. 2019;53:1-19. https://doi.org/10.1016/j. yasu.2019.04.001. 